\title{
Correction to: A source biasing and variance reduction technique for Monte Carlo radiation transport modeling of emission tomography problems
}

\author{
Seth Kilby ${ }^{1} \cdot{\text { Zhongmin } \mathrm{Jin}^{1} \cdot \text { Ashish Avachat }}^{1} \cdot$ Bryant Kanies $^{1} \cdot$ Nicolas Woolstenhulme $^{2} \cdot$ Hyoung K. Lee $^{1} \cdot$ \\ Joseph Graham ${ }^{1}$ (D)
}

Published online: 27 February 2020

๑) Akadémiai Kiadó, Budapest, Hungary 2020

\section{Correction to: Journal of Radioanalytical and Nuclear Chemistry (2019) 320:37-45 \\ https://doi.org/10.1007/s10967-019-06457-1}

In the original article, Equation 4 was incorrectly published. The correct equation is provided in this correction.

$$
F_{\mathrm{v}, \mathrm{cyl}}=\frac{2 r_{1}^{2}}{R H}+\frac{4 r_{1}^{3}}{3 R^{2} H}
$$

Since Eq. 4 was used to form Figs. 4 and 5, the conical correction factor values in Figs. 4 and 5 were overestimated. The new figures below account for the correction in Eq. 4. Note that this correction only applies to the conical approximation of the characteristic volume.
The $0.5-\mathrm{MeV}$ photopeak has an associated ratio of $0.79 \pm 0.03$. The $0.606-\mathrm{MeV}$ photopeak has a ratio of $0.80 \pm 0.08$. The 0.669 photopeak has a ratio of $0.81 \pm 0.07$. The $0.761-\mathrm{MeV}$ photopeak has a ratio of $0.79 \pm 0.03$. The $0.768-\mathrm{MeV}$ photopeak has a ratio of $0.81 \pm 0.02$. The $0.802-$ $\mathrm{MeV}$ photopeak has a ratio of $0.63 \pm 0.05$. The $1.6-\mathrm{MeV}$ photopeak has a ratio of $0.98 \pm 0.1$.

The characteristic volume correction factor for a conical geometry will underestimate the count rate of the photopeaks by approximately $18 \%$. This is still the preferred of the two characteristic volume factors as the conical factor does overestimate by approximately $45 \%$ in each of the peaks.

The original article can be found online at https://doi.org/10.1007/ s10967-019-06457-1.

Joseph Graham

grahamjose@mst.edu

1 Missouri University of Science and Technology, $301 \mathrm{~W}$ 14th St., Rolla, MO 65409, USA

2 Idaho National Laboratory, 1955 N. Fremont Avenue, Idaho Falls, ID 83415, USA 


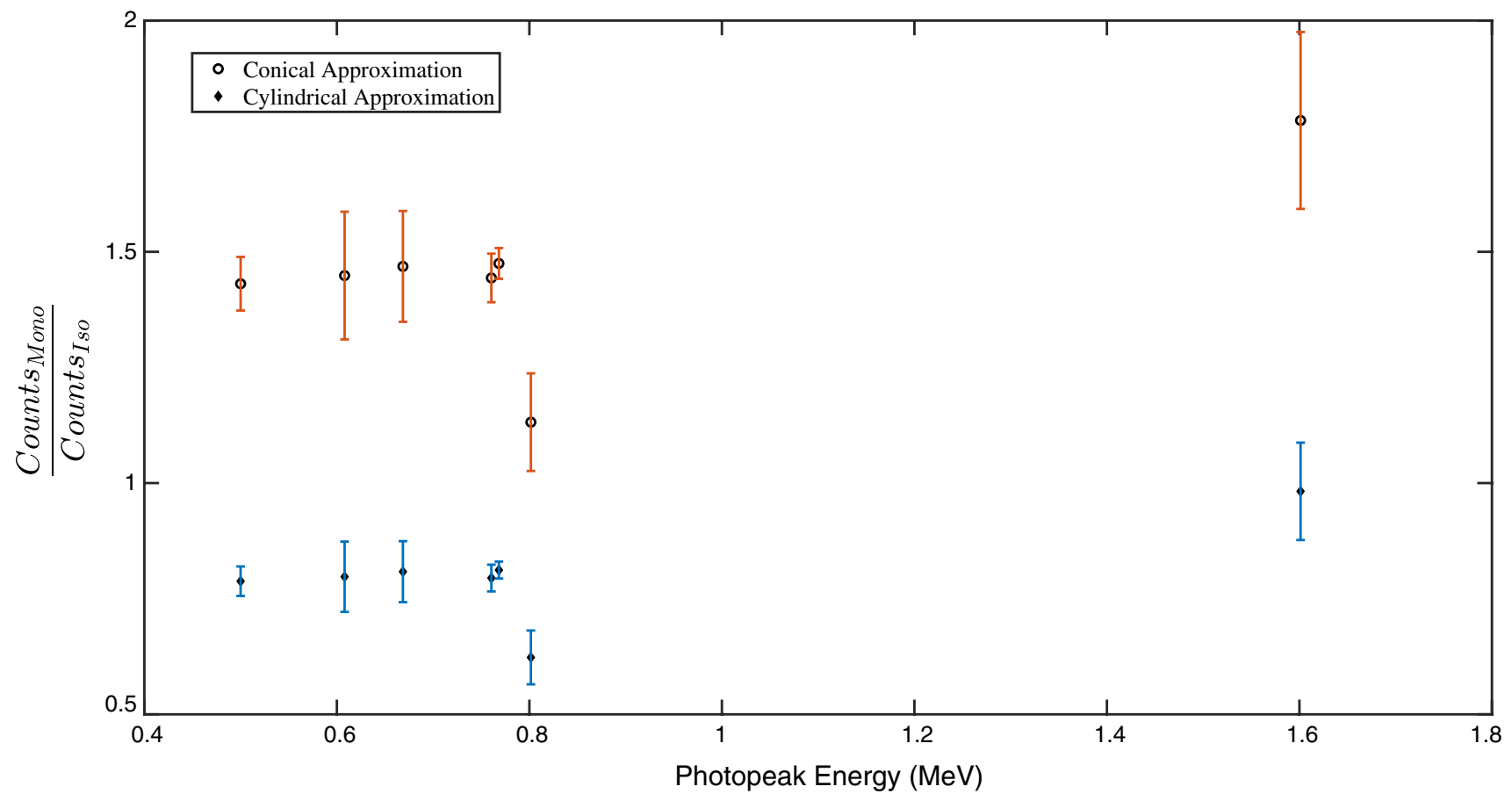

Fig. 4 Ratio of the photopeak areas for each transport method for seven high-intensity photopeaks at their corresponding decay energies. The ratios are calculated using both the truncated cone normalization and the cylindrical normalization for a 1-mm-aperture collimator

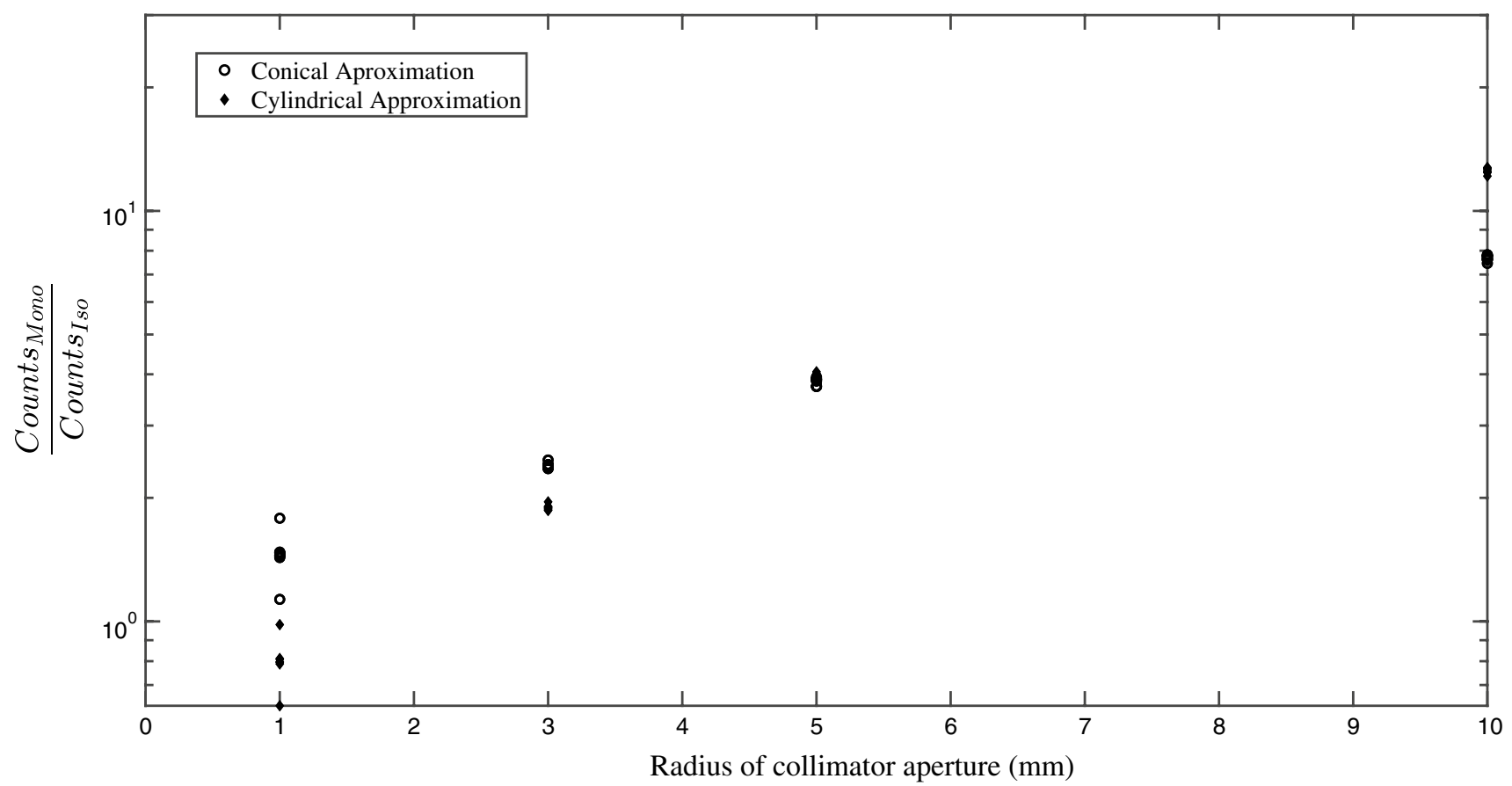

Fig. 5 Comparison between isotropic and monodirectionally biased (post-normalization) count rates as a function of aperture radii. Each data marker indicates a photopeak. Ratios for the seven strong photopeaks are shown in each cluster

Publisher's Note Springer Nature remains neutral with regard to jurisdictional claims in published maps and institutional affiliations. 